Disponível em

http://www.anpad.org.br/rac

RAC, Rio de Janeiro, v. 19, n. 1, art. 1, pp. 1-25, Jan./Fev. 2015 http://dx.doi.org/10.1590/1982-7849rac20151109

$((c)$ EY-NO

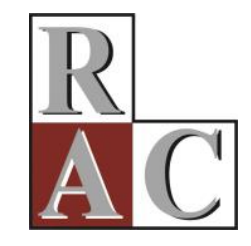

\title{
Síntese dos Principais Critérios, Métodos e Subproblemas da Seleção de Fornecedores Multicritério
}

Synthesis of Main Criteria, Methods and Issues of Multicriteria Supplier Selection 


\title{
Resumo
}

O objetivo do presente artigo é identificar na literatura quais são os principais critérios considerados no processo de decisão, os principais métodos individuais e combinados e os subproblemas da seleção de fornecedores no contexto multicritério. Cabe ressaltar que as tarefas de avaliar e identificar os melhores fornecedores para um novo produto ou serviço são essenciais ao gerenciamento da cadeia de suprimentos (GCS). Para executá-las apropriadamente, considerando-se a natureza multicritério da seleção de fornecedores, torna-se necessária a inclusão de uma abordagem de agregação dos critérios, cuja escolha depende basicamente dos objetivos da decisão, tipos de critérios a serem utilizados e racionalidade do decisor. Para atingir o objetivo proposto, optou-se por utilizar o procedimento da revisão sistemática da literatura que segue o protocolo proposto por Cronin, Ryan e Coughlan (2008), abrangendo artigos publicados nas bases ISI Web of Knowledge e Scopus, no período de 2001 a 2012. Além de sintetizar o conhecimento acerca do tema, o artigo ressalta os principais critérios, métodos e subproblemas da seleção de fornecedores, com isso, contribui ao identificar os aspectos que devem ser desenvolvidos em estudos futuros e ao apresentar aos gestores abordagens existentes para a tomada de decisões relativa à seleção de fornecedores de forma sistematizada.

Palavras-chave: alianças estratégicas; AMD; gerenciamento de fornecedores; métodos multicritério de apoio à decisão; seleção de fornecedores.

\begin{abstract}
The purpose of this paper is to identify the main criteria considered in supplier selection, the main methods used and sub-problems in a multicriteria context. The tasks of identifying the best suppliers of new products or services or even for evaluating the performance of an existing supplier is essential to supply chain management (SCM). In order to perform these tasks properly, and considering the multicriteria nature of supplier selection, it is necessary to include an approach using aggregation of criteria, primarily chosen depending on the objectives of the decision, types of criteria to be used and the rationality of the decision maker. In order to achieve this objective this research used a systematic literature review of articles published in ISI Web of Knowledge and Scopus for the period 20012012 following the protocol proposed by Cronin, Ryan and Coughlan (2008). In addition to synthesizing knowledge about the subject, the article highlights the key criteria, methods and sub-problems of selection of suppliers, contributing by identifying the aspects that should be developed in future studies. It also provides managers with existing approaches to making decisions about supplier selection in a systematic way.
\end{abstract}

Key words: strategic alliances; MCDA; supplier management; multicriteria decision aid methods; supplier selection. 


\section{Introdução}

A fim de garantir que os processos de negócios ocorram de forma eficiente, as tarefas de identificar os melhores fornecedores para um novo produto ou serviço são essenciais no GCS (Bozarth \& Handfield, 2008). No entanto muitas empresas enfrentam questões complexas quando necessitam selecionar fornecedores, pois, normalmente, esta tarefa envolve múltiplos critérios (qualitativos e quantitativos), os quais apresentam formas de mensuração diversas.

Nesse sentido, Cohen e Roussel (2004) destacam que se tornou comum a busca por ferramentas que poderiam facilitar o processo de tomada de decisão. Considerando-se a natureza que envolve múltiplos critérios na seleção de fornecedores, torna-se necessária a inclusão de uma abordagem para agregá-los adequadamente, conforme afirma Ehrlich (1996), e é importante enfatizar que existem diversos métodos utilizados para este fim. Sua escolha depende basicamente dos objetivos de decisão, tipos de critérios a serem utilizados e racionalidade do decisor.

Assim, destaca-se que o objetivo do presente artigo é identificar na literatura quais são os principais critérios considerados nesse processo de decisão, os principais métodos individuais e combinados e, também, os subproblemas comumente abordados na seleção de fornecedores no contexto multicritério.

Portanto, a fim de atingir o objetivo da pesquisa e considerando que, no campo das ciências sociais, a revisão sistemática da literatura é relativamente recente, de acordo com Velamuri, Neyer e Möslein (2011), optou-se por utilizar este procedimento metodológico, o qual segue o protocolo proposto por Cronin, Ryan e Coughlan (2008), composto pelas seguintes etapas: (a) formulação da questão de pesquisa; (b) conjunto de critérios de inclusão e exclusão; (c) seleção e acesso da literatura; (d) avaliação da qualidade da literatura incluída na revisão e; (e) análise, síntese e disseminação dos resultados. A revisão sistemática considerou artigos sobre a seleção de fornecedores no contexto multicritério, publicados nas bases ISI Web of Knowledge e Scopus, abrangendo o período de 2001 a 2012, e teve o intuito de estabelecer o estado da arte da temática abordada, bem como discutir as principais lacunas existentes na literatura com base nos artigos pesquisados.

Com base nos resultados, percebe-se um crescimento da abordagem deste tema principalmente na última década. Tais trabalhos abordam aspectos específicos e métodos individuais e combinados que se diferenciam com base em cada contexto, além disso, verificou-se que abrangem uma série de subproblemas, que não somente a seleção propriamente dita. O presente artigo, além de sintetizar o conhecimento acerca da seleção de fornecedores no contexto multicritério, ao identificar os principais critérios, métodos e subproblemas, contribui ao destacar os aspectos que podem ser desenvolvidos por pesquisadores em estudos futuros e, também, ao apresentar aos gestores as abordagens existentes para a seleção de fornecedores no contexto multicritério a fim de que as tomadas de decisão ocorram de forma sistematizada, com etapas bem-definidas e não somente baseadas na experiência.

\section{Referencial Teórico}

\section{O problema de seleção de fornecedores ou suplier selection problem (SSP)}

No GCS, a gestão do relacionamento com fornecedores define como a empresa irá interagir com seus parceiros de negócios, e isso deve ser planejado antecipadamente (Lambert, 2008). Neste subprocesso são definidos os contratos de serviços e produtos, os quais podem se basear em relacionamentos transacionais ou estratégicos (Mentzer, Min, \& Zacharia, 2000). Os relacionamentos transacionais se caracterizam por ser predominantemente competitivos e tendem a ocorrer para aquisição de produtos e serviços de menor criticidade, já os estratégicos são cooperativos e direcionados para fornecedores-chave (Bozarth \& Handfield, 2008; Lambert, 2008). 
Assim, a chave para a coordenação no GCS é uma orientação direcionada a relacionamentos próximos com fornecedores, pois atualmente não são mais empresas em particular, e sim cadeias de suprimentos que competem entre si (Mentzer et al., 2001). Outrossim, decisões de seleção de fornecedores têm se tornado estratégicas no GCS, pois as compras de fornecedores representam cerca de 40 a 60\% do preço de venda (Araz \& Ozkarahan, 2007; Sevkli, Koh, Zaim, Demirbag, \& Tatoglu, 2008). No entanto cabe ressaltar que a função compras tem incorporado um caráter estratégico devido, principalmente, ao papel dos fornecedores no processo de criação de valor da empresa (Chen, Paulraj, \& Lado, 2004).

Nesse sentido, o objetivo principal das alianças é a obtenção de melhorias na eficiência e eficácia operacional (Mentzer et al., 2000). É por meio das alianças que ocorre o compartilhamento de riscos e recompensas (Cooper, Lambert, \& Pagh, 1997). Por sua vez, destaca-se que, quando se delegam atividades para fornecedores, torna-se mais complexo o acompanhamento do desempenho destes para garantir um nível satisfatório nos processos (Araz \& Ozkarahan, 2007; Feng, Fan, \& Ma, 2010).

\section{Subproblemas relacionados à seleção de fornecedores}

O problema de seleção de fornecedores, ou Supplier Selection Problem (SSP), na língua inglesa, é bem-estabelecido na literatura e apresenta diversos subproblemas atrelados, os quais não se restringem apenas à escolha do melhor fornecedor para a aquisição de determinado produto ou serviço (Krajewski, Ritzman, \& Malhotra, 2009).

No que tange aos subproblemas do problema de seleção de fornecedores, podem ser destacados principalmente: (a) compartilhamento de processos-chave de negócios com parceiros (Cohen \& Roussel, 2004); (b) escolha de parceiros para relacionamentos colaborativos (Cohen \& Roussel, 2004); (c) aquisição de serviços ou produtos de fornecedores externos (Krajewski et al., 2009); (d) escolha de fornecedores para compartilhamento de informações (Cohen \& Roussel, 2004; Krajewski et al., 2009); (e) classificação de fornecedores em categorias a fim de direcionar a melhor estratégia de relacionamento (Cohen \& Roussel, 2004); (f) avaliação para categorização, certificação, etc. (Lambert, 2008).

Ademais, Sanayei, Mousavi e Yazdankhah (2010) ressaltam que o problema de seleção de fornecedores, sendo um dos principais processos a montante da cadeia, afeta várias áreas das empresas e torna-se uma das principais questões a ser implementada na gestão de negócios.

\section{Mensuração do desempenho dos fornecedores}

A fim de tomar decisões sobre seleção de novos fornecedores e avaliação do desempenho dos atuais, conforme Krajewski, Ritzman e Malhotra (2009), é necessário que os gestores alinhem os objetivos destas atividades com as necessidades da cadeia de suprimentos ao elaborar uma lista de critérios de desempenho a ser utilizada. Nesse sentido, S.-Y. Wang, Chang e Wang (2009) acrescentam que a mensuração do desempenho dos fornecedores possui propriedades multicritério, multidimensional e multiescala, as quais aumentam a complexidade da agregação de preferências e do processo de avaliação.

Adicionalmente, Cooper, Lambert e Pagh (1997) destacam a necessidade de se estabelecer métricas para avaliar toda a cadeia de suprimentos a fim de garantir o controle e bom andamento dos processos delegados. No mesmo sentido, Mentzer et al. (2001) ressaltaram a importância de pesquisas testando a estrutura do GCS, com base em métricas. Min e Mentzer (2004) desenvolveram uma escala de mensuração do desempenho das empresas no GCS com o propósito de testar a sua validade nomológica. Os autores encontraram indicadores de natureza estratégica que se diferenciam daqueles relacionados ao nível operacional e são considerados qualitativos, tais quais: visão e metas comuns, compartilhamento de informações, compartilhamento de riscos e recompensas, cooperação, entre outros. 
Lambert (2008) e Bozarth e Handfield (2008) corroboram os autores citados e aduzem que critérios de escolha e avaliação de fornecedores não incluem somente medidas quantitativas (como custos, taxas de entrega, entre outros), mas também fatores qualitativos, tais como estabilidade do gerenciamento; confiabilidade; cumprimento de regulamentações ambientais e potencial de relacionamento a longo prazo, etc.

Portanto a natureza do problema de seleção de fornecedores envolve múltiplos critérios, o que torna necessária a inclusão de abordagens para agregá-los adequadamente (Ehrlich, 1996). Estas abordagens são destacadas nas próximas seções.

\section{Abordagem de apoio multicritério à decisão (AMD)}

As tarefas de avaliar e selecionar fornecedores envolvem diversos critérios no processo de tomada de decisão e reconhece-se que se trata, inerentemente, de um problema de decisão multicritério (W.-P. Wang, 2010). Assim, este problema abrange a avaliação de especialistas no que tange aos vários critérios, qualitativos e quantitativos, necessários ao entendimento da situação, assim como requer métodos apropriados para a sua interpretação (Almeida, 2011).

A abordagem AMD visa fornecer aos gestores ferramentas que lhes permitam progredir na resolução de problemas de decisão, pelas quais vários objetivos, os quais frequentemente possuem pontos de vista contraditórios, devem ser levados em consideração, como é o caso, por exemplo, da dicotomia existente entre reduzir custos e aumentar a qualidade (Almeida, 2011). Considerando a natureza imperfeita dos julgamentos e aspirações humanos, nem sempre o mais adequado é selecionar uma decisão baseada em um critério único de avaliação, como é o caso do custo (Roy, 1996). Basicamente, a maioria dos problemas de decisão de interesse prático possui ao menos duas alternativas e dois critérios, portanto é necessário analisá-los em termos dos objetivos e critérios que os privilegiam (Almeida, 2011; Roy, 1996).

Esses objetivos considerados simultaneamente, por sua vez, são associados a variáveis que os representem e permitam que cada alternativa seja avaliada com base em cada objetivo, essas variáveis são os critérios (Almeida, 2011; Ehrlich, 1996; Roy, 1996).

\section{Principais abordagens e métodos AMD}

A fim de que a avaliação das alternativas mediante os critérios seja agregada e a tomada de decisão seja facilitada, podem ser utilizados métodos da abordagem AMD. Bozarth e Handfield (2008) destacam que esses métodos são muito úteis quando existe um mix de critérios qualitativos e quantitativos e um grande número de alternativas a serem consideradas no processo de seleção e avaliação de fornecedores, pois eles auxiliam a formalizar e estruturar a tomada de decisão.

No entanto a escolha da abordagem a qual o método está vinculado precede sua escolha. Vincke (1992) e Roy (1996) concordam que os especialistas em AMD, geralmente, dividem as abordagens em: (a) Teoria da utilidade multiatributo ou do Critério único de síntese; (b) Abordagem de sobreclassificação ou superação; e, (c) Métodos interativos, os quais são descritos na Tabela 1: 
Tabela 1

\section{Síntese das Abordagens AMD}

\begin{tabular}{|c|c|}
\hline ABORDAGENS & DESCRIÇÃO \\
\hline $\begin{array}{l}\text { Teoria da Utilidade } \\
\text { Multiatributo ou do } \\
\text { Critério Único de } \\
\text { Síntese }\end{array}$ & $\begin{array}{l}\text { Deriva-se da corrente de pensamento americana, as preferências do decisor por } \\
\text { determinada alternativa, quando a avalia mediante um conjunto de critérios ou } \\
\text { indicadores, são agregadas em um valor de utilidade único, que é mesurado de uma forma } \\
\text { aditiva (com trade-offs), ou seja, é gerado um score de cada alternativa com base no } \\
\text { desempenho que apresentou em cada critério, assim as alternativas melhor avaliadas são } \\
\text { as que obtiverem o melhor score (Almeida, 2011). Alguns métodos desta abordagem } \\
\text { podem ser citados: MAUT, SMART, TOPSIS, AHP. }\end{array}$ \\
\hline
\end{tabular}

Sobreclassificação ou Derivada da corrente francesa, o principal objetivo é a construção de relações binárias que Subordinação representem as preferências do decisor com base na informação disponível (sem trade$o f f s$ ) entre critérios, neste caso, não se obtém um score das alternativas mediante seu desempenho em cada critério (Léger \& Martel, 2002). Por meio de uma comparação par a par, verifica-se qual alternativa é superior em cada critério, estabelecendo-se uma relação de superação no confronto entre duas alternativas. Assim, é melhor avaliada a alternativa que apresentar superioridade na maioria dos critérios, esta abordagem é baseada no sistema de eleição de Condorcet e é considerada uma abordagem mais equilibrada, tendo em vista que é escolhida a alternativa que possuir um desempenho satisfatório na maioria dos critérios, segundo Almeida (2011). Os principais métodos desta abordagem são os das famílias: ELECTRE, PROMETHÉE.

Métodos Interativos São desenvolvidos principalmente no âmbito da Multi-objective Linear Programming (MOLP), os quais se caracterizam por possuir passos computacionais e serem interativos, permitindo trade-offs (Léger \& Martel, 2002). Os métodos da MOLP buscam uma alternativa que seja claramente superior em todos os objetivos estabelecidos (dominante), para isso, efetuam a agregação das preferências dos decisores e cálculos matemáticos, interativos e sucessivos, avaliação destas soluções, de possível alteração da estrutura de preferências em face da nova informação disponível, com vista à convergência para uma solução final que estabeleça um compromisso aceitável entre as funções objetivo (Antunes \& Alves, 2012). Cabe ressaltar que este processo é diferente das abordagens do critério único de síntese e de sobreclassificação, as quais, mediante a comparação entre critérios, buscam a solução mais satisfatória e não a dominante (Almeida, 2011). Alguns métodos dessa abordagem podem ser citados: STEM, TRIMAP, ICW, PARETO RACE (Antunes \& Alves, 2012).

Nota. Fonte: Adaptado de Léger, J., \& Martel, J.-M. (2002). A multicriteria assignment procedure for a nominal sorting problematic (pp. 349-352). European Journal of Operational Research, 138(2), 349-364. doi: 10.1016/S0377-2217(01)00251X; Almeida, A. T. de (2011). O conhecimento e o uso de métodos multicritério de apoio a decisão (pp. 22-25). Recife: Editora Universitária da UFPE; Antunes, C., \& Alves, M. (2012, setembro). Programação linear multiobjetivo-métodos interativos e software (pp. 4726-4727). Anais do Congreso Latino-Iberoamericano de Investigación Operativa, Rio de Janeiro, RJ, Brasil, 16.

A escolha das abordagens apresentadas na Tabela 1 dependerá principalmente da racionalidade do decisor ao demonstrar suas preferências, as quais podem ser basicamente: (a) Aditiva: as alternativas são avaliadas mediante cada critério, o desempenho das alternativas é avaliado quantitativamente (valores numéricos) ou qualitativamente (por meio de escalas) e é estabelecido um score para cada alternativa. Essa racionalidade permite trade-offs entre critérios, o que significa afirmar que uma alternativa com um péssimo desempenho em uma alternativa (por exemplo: qualidade) pode ser compensada por um ótimo desempenho em outro critério (por exemplo: custo); e, (b) Não aditiva: as alternativas são avaliadas em pares pelas relações: preferência forte $(\mathrm{P}+)$, preferência fraca $(\mathrm{P}-)$, indiferença (I) ou incomparabilidade (R), no lugar de números. Neste caso, não é gerado um score para cada alternativa, este sistema é similar ao utilizado nas Olimpíadas (medalhas de ouro, prata e bronze), pelo qual as alternativas são avaliadas segundo a quantidade e qualidade dos votos que obtiveram, ou seja, não permite trade-offs entre critérios (Almeida, 2011; Vincke, 1992). Na Tabela 2, estão elencados os principais métodos utilizados em cada abordagem: 
Tabela 2

Principais Métodos Provenientes das Abordagens AMD

\begin{tabular}{ll}
\hline MÉTODO & DESCRIÇÃO \\
\hline ABORDAGEM DA TEORIA DA UTILIDADE MULTIATRIBUTO/CRITÉRIO ÚNICO DE SÍNTESE \\
\hline $\begin{array}{l}\text { Multiple Attribute } \\
\text { Function (MAUT) }\end{array}$ & $\begin{array}{l}\text { Baseia-se nos conceitos de modelagem de preferência tradicional, admite trade-offs } \\
\text { entre critérios e somente duas situações: Preferência estrita (P) e Indiferença (I). É } \\
\text { construída uma função utilidade de agregação U (g1, ..., gi, ..., gn), agregando os } \\
\text { critérios gj (j }=1, \ldots, \mathrm{n}) \text { em um critério único de síntese, explicitando as preferências } \\
\text { do decisor. Ordena as alternativas. }\end{array}$
\end{tabular}

Simple Multicriteria- É uma simplificação da MAUT, julga a avaliação das alternativas considerando o Atribute Rating Tecnique pior e melhor estímulos, faz uso da estratégia da aproximação heroica para justificar (SMART) aproximações lineares das funções utilidade multidimensional. A agregação das preferências do decisor com base nas alternativas e critérios é aditiva, ou seja, considera trade-offs entre critérios. É um método de ordenação de alternativas. Derivação: SMARTS (com swing de pesos).

Technique for Order Avalia o desempenho das alternativas através da similaridade desta com a solução Preference by Smilarity to ideal, assim a melhor alternativa seria aquela que é a mais próxima da solução ideal Ideal Solution (TOPSIS) positiva e a mais distante da solução ideal negativa. A solução ideal positiva sendo aquela que maximiza os critérios de benefício e minimiza os critérios de custo; já a solução ideal negativa maximiza os critérios de custo e minimiza os critérios de benefício. É um método de ordenação de alternativas e permite trade-offs entre critérios

Analytic Hierarchy Decompõe o problema em diversos em fatores, com relações entre si, por meio da Process (AHP) construção de uma hierarquia, possibilita ao decisor definir prioridades e realizar o julgamento de preferências entre alternativas, comparando-as em pares para cada critério por meio de matrizes e baseados na escala numérica de Saaty. É um método de ordenação das alternativas e considera trade-offs entre critérios.

\begin{tabular}{|c|c|}
\hline ABORDAGEM DE SOB & CLASSIFICA \\
\hline $\begin{array}{l}\text { Elimination and Choice } \\
\text { Translating Algorithm } \\
\text { (ELECTRE) }\end{array}$ & $\begin{array}{l}\text { Compreende dois procedimentos principais: I) construção de uma ou várias relações } \\
\text { de sobreclassificação/superação; e, II) exploração desta(s) relação(s). A construção } \\
\text { de uma relação ou mais relações de sobreclassificação tem como objetivo comparar } \\
\text { cada par de ações. Não permite trade-offs entre critérios. Derivações: ELECTRE I e } \\
\text { IS (escolha e ordenação); ELECTRE II, III e IV (ordenação); ELECTRE TRI } \\
\text { (classificação). }\end{array}$ \\
\hline $\begin{array}{l}\text { Preference Ranking } \\
\text { Method for Enrichment } \\
\text { Evaluation } \\
\text { (PROMETHEE) }\end{array}$ & $\begin{array}{l}\text { Consiste em realizar a comparação das alternativas em pares e construir uma relação } \\
\text { de sobreclassificação de valores, destacando-se por buscar envolver conceitos e } \\
\text { parâmetros que têm interpretação física ou econômica. Não permite trade-offs entre } \\
\text { critérios. Derivações: PROMETHEE II (ordenação); PROMETHEE III e IV } \\
\text { (ordenação); PROMETHEE V (ordenação); PROMETHEE VI (ordenação, } \\
\text { PROMSORT (classificação). }\end{array}$ \\
\hline
\end{tabular}

\section{ABORDAGEM DOS MÉTODOS INTERATIVOS}

Step Method (STEM) Em cada fase de cálculo é calculada a solução que minimiza uma distância pesada de Chebyshev à solução ideal, que é colocada à apreciação do decisor na fase de diálogo seguinte. Se os valores das funções objetivo são todos considerados satisfatórios, o processo termina. Caso contrário, o decisor estabelece que funções objetivo aceita relaxar e qual o valor desta relaxação, para tentar melhorar os outros objetivos que ainda não têm valores satisfatórios. É um método de escolha e ordenação de alternativas, considera trade-offs. 
Tabela 2 (continuação)

\begin{tabular}{|c|c|}
\hline MÉTODO & DESCRIÇÃO \\
\hline $\begin{array}{l}\text { Interval Criterion Weights } \\
\text { (ICW) }\end{array}$ & $\begin{array}{l}\text { O decisor escolhe uma solução numa amostra de soluções não dominadas que lhe é } \\
\text { apresentada em cada fase de diálogo. Em cada fase de cálculo são otimizadas várias } \\
\text { somas ponderadas das funções objetivo, com combinações de pesos regularmente } \\
\text { dispersas no diagrama paramétrico, o que evita requerer ao decisor a indicação } \\
\text { explícita de pesos. É um método de escolha de alternativas, considera trade-offs. }\end{array}$ \\
\hline Pareto Race & $\begin{array}{l}\text { Realiza uma pesquisa direcional livre sobre a região não dominada. A informação } \\
\text { de preferências consiste na indicação das funções objetivo a melhorar, o que provoca } \\
\text { a alteração a direção da pesquisa. As soluções são calculadas definindo uma direção } \\
\text { que oferece uma variação nos valores das funções objetivo que está de acordo com } \\
\text { as preferências do decisor, a qual é depois projetada sobre a região não dominada. É } \\
\text { um método de escolha e ordenação de alternativas, considera trade-offs. }\end{array}$ \\
\hline $\begin{array}{l}\text { Tricriterion } \begin{array}{l}\text { Multiobjective } \\
\text { Linear } \quad \text { Programming } \\
\text { (TRIMAP) }\end{array}\end{array}$ & $\begin{array}{l}\text { Efetua uma pesquisa livre no sentido de uma aprendizagem progressiva e seletiva do } \\
\text { conjunto de soluções não dominadas, combinando a redução da região admissível } \\
\text { com a redução do diagrama paramétrico. Em cada fase de cálculo é otimizada uma } \\
\text { soma ponderada das funções objetivo. O decisor pode especificar limitações } \\
\text { inferiores para funções objetivo, que são traduzidas para o diagrama paramétrico, e } \\
\text { impor restrições diretamente nos pesos. É um método de escolha e ordenação de } \\
\text { alternativas, considera trade-offs. }\end{array}$ \\
\hline
\end{tabular}

Nota. Fonte: Adaptado de Léger, J., \& Martel, J.-M. (2002). A multicriteria assignment procedure for a nominal sorting problematic (pp. 349-352). European Journal of Operational Research, 138(2), 349-364. doi: 10.1016/S0377-2217(01)00251X; Almeida, A. T. de (2011). O conhecimento e o uso de métodos multicritério de apoio a decisão (pp. 22-25). Recife: Editora Universitária da UFPE; Antunes, C., \& Alves, M. (2012, setembro). Programação linear multiobjetivo-métodos interativos e software (pp. 4726-4727). Anais do Congreso Latino-Iberoamericano de Investigación Operativa, Rio de Janeiro, RJ, Brasil, 16.

No entanto, com relação à escolha dos métodos relacionados na Tabela 2, cabe ressaltar que a situação do problema a influencia, pois as decisões que envolvem o contexto multicritério podem ser direcionadas por várias problemáticas: (a) Escolha: reduzir a um conjunto menor o número de alternativas existentes; (b) Ordenação: ordenar as alternativas em ordem crescente ou decrescente; e, (c) Classificação: categorizar as alternativas em grupos por similaridade, de forma categórica ou ordenada (Roy, 1996; Vincke, 1992). Ademais, acrescenta-se que existem naturezas de critérios diferentes, os quais podem ser classificados basicamente em quantitativos e qualitativos. Alguns métodos lidam bem com critérios quali e quantitativos, como é o caso do AHP, Electre e Promethée, sendo a MAUT e os métodos interativos mais adaptados a critérios quantitativos.

Há ainda que se acrescentar a essas abordagens os métodos da Teoria Fuzzy, os quais são frequentemente agregados aos métodos AMD devido à sua capacidade de modelar os processos subjetivos de avaliação humana, convertendo essas avaliações em escalas de números fuzzy (Larbani, Huang, \& Tzeng, 2011).

Porquanto não existe um consenso dos profissionais de AMD sobre o melhor método a ser utilizado e é importante enfatizar que a escolha de um método deve levar em consideração a situação de decisão que envolve os objetivos, a problemática escolhida (escolha, ordenação, classificação), os tipos de critérios (quantitativos e/ou qualitativos) e a racionalidade do decisor que influenciará no tipo de agregação das preferências relativas às alternativas e aos critérios (aditiva ou não aditiva). Desta forma, ao longo dos anos, vários métodos individuais e combinados têm surgido (Almeida, 2011; Araz \& Ozkarahan, 2007). Desse modo, após elencadas as principais características do problema de seleção de fornecedores e também da abordagem AMD, é possível apresentar o procedimento metodológico utilizado na pesquisa, o qual é descrito na próxima seção. 


\section{Procedimento Metodológico Utilizado na Pesquisa}

Para determinar o estudo da arte da pesquisa, com o intuito de identificar os principais critérios, métodos e subproblemas relativos ao problema de seleção de fornecedores no contexto multicritério, utilizou-se o procedimento técnico da revisão da literatura, o qual possui basicamente duas perspectivas: (a) revisões de tópicos que possuem um conhecimento acumulado, o qual requer uma análise e síntese; e, (b) revisões de assuntos emergentes, cuja contribuição é a exposição de fundações teóricas potenciais, a qual normalmente não é tão extensa como a primeira (Webster \& Watson, 2002). O tema pesquisado neste artigo se enquadra na primeira perspectiva de revisão da literatura.

Outrossim, relacionado ao procedimento técnico da pesquisa, existem também dois tipos de revisão da literatura, conforme ressaltam Cronin et al. (2008): (a) revisão narrativa ou tradicional da literatura; e, (b) revisão sistemática da literatura. O primeiro tipo sumariza a literatura, embora não deixe explícito ao leitor os critérios utilizados para a seleção das fontes. Já a revisão sistemática usa uma abordagem bem-definida para revisar a literatura, a qual segue um protocolo para selecionar e analisar as fontes (Cronin, Ryan, \& Coughlan, 2008). Cabe também ressaltar que as revisões sistemáticas da literatura podem ser ancoradas em abordagens qualitativas ou quantitativas (meta-análise), a depender dos objetivos da pesquisa (De-la-Torre-Ugarte-Guanilo, Takahashi, \& Bertolozzi, 2011).

Com relação aos artigos de revisão de literatura acerca da seleção de fornecedores podem ser destacados os trabalhos de Weber, Current e Benton (1991), Degraeve, Labro e Roodhooft (2000) e Boer, Labro e Morlacchi (2001). No entanto é importante enfatizar que estes três artigos utilizaram o procedimento da revisão tradicional ou narrativa da literatura, nos quais não fica claro qual foi o protocolo para seleção de artigos a serem analisados.

Já no caso da revisão sistemática da literatura, esta é extensivamente utilizada no campo da medicina em testes clínicos (De-la-Torre-Ugarte-Guanilo, Takahashi, \& Bertolozzi, 2011; Higgins \& Green, 2011). No campo das ciências sociais, este tipo de procedimento é relativamente recente (Velamuri, Neyer, \& Möslein, 2011). Todavia algumas pesquisas podem ser destacadas: sobre o GCS (Wong, Tjosvold, \& Zhang, 2005); sobre a seleção de fornecedores para terceirização (Khan, Niazi, \& Ahmad, 2011); sobre a seleção de fornecedores de software (Wen, Li, Lin, Hu, \& Huang, 2012).

Cronin et al. (2008) propuseram um protocolo de revisão sistemática baseado naquele utilizado na área de medicina e proposto por Higgins e Green (2011), envolvendo as etapas: (a) formulação da questão de pesquisa; (b) conjunto de critérios de inclusão e exclusão; (c) seleção e acesso da literatura; (d) avaliação da qualidade da literatura incluída na revisão; e, (e) análise, síntese e disseminação dos resultados. Assim, baseado neste protocolo, apresenta-se a síntese do procedimento na Figura 1. 


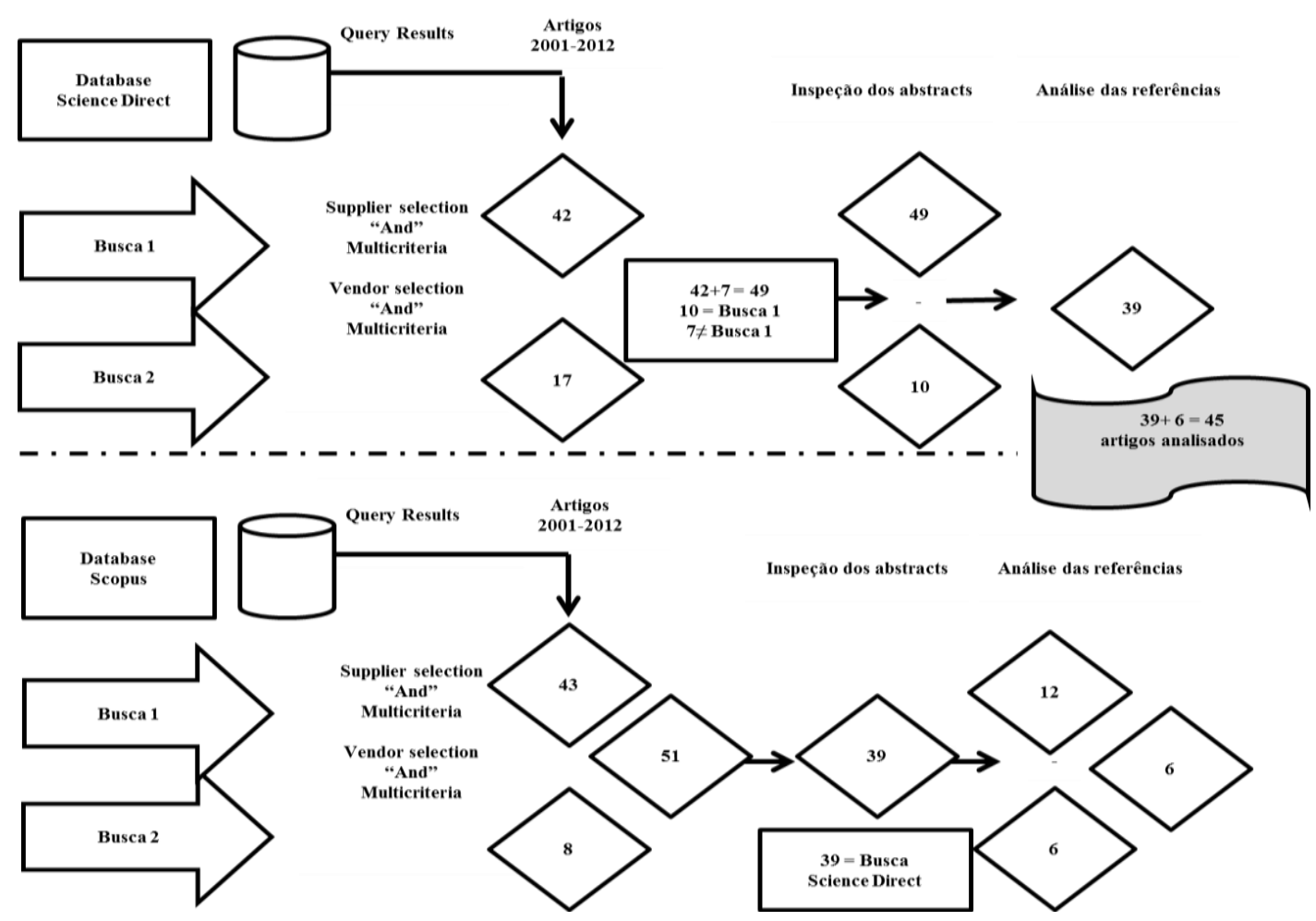

Figura 1. Protocolo da Revisão Sistemática da Literatura.

A Figura 1 sintetiza o protocolo da revisão sistemática da literatura realizada para atingir o objetivo deste artigo, composto pelas etapas descritas a seguir.

1. Definição da questão da pesquisa: Quais são os principais critérios, métodos e subproblemas da seleção de fornecedores no contexto multicritério?

2. Definição do conjunto de critérios de inclusão e exclusão: Os critérios abrangem as bases científicas selecionadas, período de publicação, tipos de artigos, palavras-chave e operadores booleanos. Devido ao fato de que o tema seleção de fornecedores é multidisciplinar e pode ser encontrado em periódicos de diversas áreas - Análise de Decisões; Administração; Engenharia da Produção; Engenharia Civil; Engenharia Mecânica; Engenharia de Software; Tecnologia da Informação; Economia; Ciências Contábeis; Matemática; entre outras, definiu-se que a pesquisa ocorreria nas bases científicas: (a) ISI Web of Knowledge; e, (b) Scopus, as quais são multidisciplinares e abrangem artigos de periódicos com alto fator de impacto e de todas as áreas supracitadas. Após a definição das bases, estabeleceu-se o período de publicação, o qual abrange os artigos publicados de 2001 a 2012. Quanto às palavras-chave, foram definidas com base na questão da pesquisa, as palavras: supplier selection e, seu sinônimo, vendor selection, ademais, tendo em vista o contexto envolvendo múltiplos critérios, definiu-se a palavra: multicriteria. No tocante aos tipos de artigos, definiu-se que seriam abrangidos somente artigos completos publicados em periódicos, o que excluiu artigos publicados em anais de eventos, patentes e capítulos de livros. No que se refere aos operadores booleanos utilizados, optou-se pelo operador and, excluindo-se os operadores not e or, considerando que os resultados deveriam refletir artigos sobre seleção de fornecedores no contexto multicritério.

3. Seleção e acesso da literatura: Conforme citado anteriormente, a localização dos artigos ocorreu por meio da busca nas bases ISI Web of Knowledge e SCOPUS. Na primeira base, utilizando-se do operador booleano and, foram identificados 42 artigos com as palavras-chave Supplier Selection, Multicriteria e, especificando o período de publicação 2001-2012, então, realizou-se uma segunda pesquisa alterando-se as palavras-chave de Supplier Selection para Vendor Selection, mantendo-se as demais e obteve-se um resultado de 17 artigos, dos quais apenas sete se diferenciaram da primeira busca. Na base SCOPUS, foram identificados 43 artigos com as palavras-chave Supplier Selection 
e, alterando-se para Vendor Selection, obteve-se oito, totalizando 51 artigos, dos quais 39 coincidiram com os apresentados pela base ISI Web of Knowledge e outros 12 se diferenciaram.

4. Avaliação da qualidade da literatura incluída na revisão: Na base ISI Web of Knowledge, foram identificados 49 artigos, dos quais 39 foram selecionados para análise, 10 foram eliminados por não se encaixarem adequadamente no tema de estudo, após a análise dos abstracts e introdução. Com relação aos artigos da base Scopus, dos 12 artigos encontrados, constatou-se que apenas seis se encaixavam no tema da pesquisa, os outros seis foram eliminados por se distanciarem do tema seleção de fornecedores com foco multicritério. Assim, o total de artigos analisados foi de 45 . Portanto foram realizados o fichamento e compilação dos dados dos 45 artigos selecionados, destacando-se os aspectos relacionados à questão da pesquisa previamente definida na etapa a, envolvendo: autores; período de publicação; subproblema relativo à seleção de fornecedores; os principais critérios utilizados e os métodos que consideram múltiplos critérios (individuais e combinados).

5. Análise, síntese e disseminação dos resultados: Esta fase abrangeu a análise detalhada de cada artigo, necessária para a análise e interpretação dos trabalhos publicados. Dessa forma, foram elaboradas tabelas com os principais critérios utilizados e respectivos autores; os principais subproblemas da seleção de fornecedores; as principais abordagens no contexto multicritério, sendo que estas foram classificadas por abordagem. Assim, os resultados foram analisados, possibilitando sua discussão na próxima seção e também a identificação de lacunas na literatura, as quais podem ser desenvolvidas em estudos futuros.

\section{Apresentação dos Resultados: Estado da Arte Sobre Seleção de Fornecedores}

Esta seção apresenta os resultados dos artigos selecionados para a análise, considerando-se o protocolo de revisão sistemática estabelecido na seção 3. Assim, foram evidenciados os principais critérios abordados, os principais subproblemas de seleção de fornecedores e os métodos comumente utilizados, categorizados nas abordagens AMD e ainda as variações da Lógica Fuzzy e outros métodos provenientes de outras abordagens.

Cabe ressaltar que um dos primeiros e mais citados estudos em relação ao problema de seleção de fornecedores no contexto multicritério foi publicado em 1966, por Dickson. O autor propôs que os fornecedores devem ser selecionados usando 23 critérios, os seis considerados mais importantes foram: Qualidade; Entrega; Histórico de desempenho; Políticas de garantia; Capacidade produtiva e Preço. Outro trabalho relevante a ser citado é o de Ellram (1990), que propôs alguns critérios, chamados soft factors, os quais incluem fatores considerados na construção de alianças estratégicas entre compradores e fornecedores nas cadeias de suprimentos. A aplicabilidade desses critérios depende do produto ou serviço e do mercado para o qual serão direcionados, conforme afirmam Sevkli, Koh, Zaim, Demirbag e Tatoglu (2008).

Weber et al. (1991) revisaram 74 artigos, desde 1966, com o intuito de identificar os principais critérios e métodos analíticos usados para a seleção de fornecedores, quanto aos critérios, os cinco mais citados foram: Qualidade, Entrega, Preço da rede, Localização geográfica e Facilidades de produção. Quanto às abordagens encontradas, podem ser categorizadas em: (a) Modelos de ponderação linear; (b) Modelos com programação matemática; e, (c) Abordagens probabilísticas e estatísticas. Degraeve et al. (2000) indicou o uso do conceito de Total Cost of Ownership (TCO) como base de comparação dos modelos de seleção de fornecedores, os quais podem ser categorizados em: (a) Métodos de ponderação linear, (b) Abordagens de custo total; (c) Programação matemática; e, (d) Estatísticos.

Em seguida, Boer et al. (2001) revisaram os artigos com modelos de seleção de fornecedores e identificaram as fases em que estes modelos foram utilizados: (a) Definição do problema, (b) Formulação dos critérios; (c) Qualificacão; (d) Escolha; sendo as três primeiras fases pouco abordadas 
na literatura. Os autores encontraram como principais abordagens: (a) Métodos categóricos, (b) Data envelopment analysis (DEA); (c) Cluster analysis (CA); (d) Total cost of ownership (TCO); (e) Modelos de programação matemática; (f) Modelos estatísticos, e, (g) Modelos de inteligência artificial.

Na Tabela 3 discriminam-se os critérios mais utilizados encontrados na presente pesquisa, em ordem decrescente de utilização.

Tabela 3

Critérios Utilizados na Seleção e Avaliação de Fornecedores

\begin{tabular}{ll}
\hline CRITÉRIOS & AUTORES \\
\hline Custo & Almeida (2001, 2007); Mikhailov (2002); Kumar, Vrat e Shankar (2004); \\
& Simatupang, Wright e Sridharan (2004); Choy, Lee, Lau e Choy (2005); Pi e \\
& Low (2005, 2006); Chang, Wang e Wang (2006); Amid, Ghodsypour e O’Brien \\
& (2006, 2009, 2011); Araz e Ozkarahan (2007); Araz, Ozfirat e Ozkarahan (2007); \\
& Chou e Chang (2008); Ting e Cho (2008); Ustun e Demirtas (2008); Yang, Chiu, \\
& Tzeng e Yeh (2008); Ng (2008); Razmi, Songhori e Khakbaz (2009); Ordoobadi \\
& (2009a, 2009b); Boran, Genç, Kurt e Akay (2009); Li e Zabinsky (2011); Ku, \\
& Chang e Ho (2009); Lin (2009); Zhang, Zhang, Lai e Lu (2009); S.-Y. Wang et \\
& al. (2009); Park, Shin e Chang (2010); W.-P. Wang (2010); Keskin, Ilhan e \\
& Özkan (2010); Sanayei et al. (2010), Liao e Kao (2010); Khaleie, Fasanghari e \\
& Tavassoli (2012); Yayla, Yildiz e Ozbek (2012).
\end{tabular}

Qualidade

Entrega on-time

Serviço

Estabilidade financeira

Capacidade tecnológica

Entregas atrasadas

Facilidade de comunicação

Resposta às exigências dos Almeida (2007); Ku et al. (2009); S.-Y. Wang et al. (2009); Chang et al. (2006); clientes

Mikhailov (2002); Dulmin e Mininno (2003); Pi e Low (2005, 2006); Choy et al. (2005); Chang et al. (2006); Udin, Khan e Zairi (2006); Amid et al. (2006, 2011); Araz e Ozkarahan (2007); Araz et al. (2007); Sevkli et al. (2008); Ha e Krishnan (2008); Chou e Chang (2008); Ting e Cho (2008); Yang et al. (2008); Ng (2008); Ustun e Demirtas (2008); Ordoobadi (2009b); Razmi et al. (2009); Lin (2009); Zhang et al. (2009); Boran et al. (2009); Ku et al. (2009); W.-P. Wang (2010); Keskin et al. (2010); Sanayei et al. (2010); Park et al. (2010); Liao e Kao (2010); Khaleie et al. (2012); Yayla et al. (2012).

Choy et al. (2005); Pi e Low (2005, 2006); Almeida (2007); Lee, Kwon e Severance (2007); Araz e Ozkarahan (2007); Araz et al. (2007); Ha e Krishnan (2008); Chou e Chang (2008); Ng (2008); Ku et al. (2009); Lin (2009); Zhang et al. (2009); Boran et al. (2009); Ordoobadi (2009b); Liao e Kao (2010); W.-P. Wang (2010); Sanayei et al. (2010); Park et al. (2010); Khaleie et al. (2012); Yayla et al. (2012).

Simatupang et al. (2004); Pi e Low (2005, 2006); Chang et al. (2006); Amid et al. (2006, 2011); Ting e Cho (2008); Ustun e Demirtas (2008); Ku et al. (2009); Zhang et al. (2009); S.-Y. Wang et al. (2009); Ordoobadi (2009a); Liao e Kao (2010).

Mikhailov (2002); Udin et al. (2006); Araz e Ozkarahan (2007); Araz et al. (2007); Amin e Razmi (2009); Liao e Kao (2010); Feng et al. (2010); Keskin et al. (2010); Park et al. (2010).

Dulmin e Mininno (2003); Choy et al. (2005); Araz e Ozkarahan (2007); Yang et al. (2008); Feng et al. (2010); Sanayei et al. (2010); Park et al. (2010); Khaleie et al. (2012).

Kumar et al. (2004); Ustun e Demirtas (2008); Amid et al. (2009); Lin (2009); Razmi et al. (2009).

Choy et al. (2005); Araz e Ozkarahan (2007); Ustun e Demirtas (2008); Ku et al. (2009); Park et al. (2010).

Ordoobadi (2009a). 


\section{Tabela 3 (continuação)}

\begin{tabular}{|c|c|}
\hline CRITÉRIOS & AUTORES \\
\hline Flexibilidade & $\begin{array}{l}\text { Keskin et al. (2010); Sanayei et al. (2010); Razmi et al. (2009); Choy et al. } \\
\text { (2005); Kumar et al. (2004); Yayla et al. (2012). }\end{array}$ \\
\hline Gerenciamento e organização & $\begin{array}{l}\text { Araz et al. (2007); Ha e Krishnan (2008); Amin e Razmi (2009); Park et al. } \\
\text { (2010). }\end{array}$ \\
\hline $\begin{array}{l}\text { Facilidades e capacidade de } \\
\text { produção }\end{array}$ & $\begin{array}{l}\text { Sevkli et al. (2008); Keskin et al. (2010); Amid et al. (2009); Kumar et al. } \\
\text { (2004); Park et al. (2010). }\end{array}$ \\
\hline Suporte & $\begin{array}{l}\text { Araz e Ozkarahan (2007); Yang et al. (2008); Ustun e Demirtas (2008); Ku et al. } \\
\text { (2009); Amin e Razmi (2009). }\end{array}$ \\
\hline Culturas compatíveis & Choy et al. (2005); Chou e Chang (2008); Feng et al. (2010). \\
\hline Localização geográfica & $\begin{array}{l}\text { Ng (2008); Ku et al. (2009); Keskin et al. (2010); Park et al. (2010); Yayla et al. } \\
\text { (2012). }\end{array}$ \\
\hline $\begin{array}{l}\text { Capacidades técnicas e } \\
\text { organizacionais }\end{array}$ & Bottani e Rizzi (2008); Ku et al. (2009); Lin (2009); Keskin et al. (2010). \\
\hline $\mathrm{P} \& \mathrm{D}$ & $\begin{array}{l}\text { Simatupang et al. (2004); Choy et al. (2005); Zhang et al. (2009); Boran et al. } \\
\text { (2009). }\end{array}$ \\
\hline Confiança mútua & Ustun e Demirtas (2008); Govindan, Kannan e Haq (2010); Park et al. (2010). \\
\hline
\end{tabular}

O levantamento dos critérios, apresentado na Tabela 3, não inclui os trabalhos de revisão da literatura, mas somente aqueles que propõem e utilizam métodos multicritério para a seleção e avaliação de fornecedores e para isso elegem critérios específicos. Foram destacados os 18 critérios, citados por três ou mais artigos. Ademais, na Tabela 4, é possível visualizar os diferentes subproblemas no âmbito de seleção de fornecedores, também, por ordem decrescente de utilização.

Tabela 4

\section{Subproblemas de Seleção de Fornecedores}

\begin{tabular}{|c|c|}
\hline Subproblemas Abordados na Literatura & Autores \\
\hline $\begin{array}{l}\text { Seleção e Avaliação do desempenho dos fornecedores } \\
\text { para compra de componentes/materiais }\end{array}$ & $\begin{array}{l}\text { Mikhailov (2002); Dulmin e Mininno (2003); Pi e Low } \\
\text { (2005); Amid et al. (2006); Sevkli et al. (2008); Ha e } \\
\text { Krishnan (2008); Ng (2008); Yang et al. (2008); Boran } \\
\text { et al. (2009); Ordoobadi (2009a); Liao e Kao (2010); } \\
\text { Sanayei et al. (2010); Yayla et al. (2012); Khaleie } \text { et al. } \\
\text { (2012). }\end{array}$ \\
\hline $\begin{array}{l}\text { Seleção de fornecedores e alocação ótima de pedidos, } \\
\text { satisfazendo restrições }\end{array}$ & $\begin{array}{l}\text { Araz et al. (2007); Ustun e Demirtas (2008); Razmi et } \\
\text { al. (2009); Lin (2009); Amid et al. (2009); Li e } \\
\text { Zabinsky (2011). }\end{array}$ \\
\hline $\begin{array}{l}\text { Gerenciamento de fornecedores, incluindo a seleção, } \\
\text { avaliação e desenvolvimento }\end{array}$ & $\begin{array}{l}\text { Araz e Ozkarahan (2007); Araz et al. (2007); Amin e } \\
\text { Razmi (2009); Govindan et al. (2010). }\end{array}$ \\
\hline $\begin{array}{l}\text { Avaliação do desempenho de fornecedores e } \\
\text { classificação em categorias de acordo com suas } \\
\text { similaridades }\end{array}$ & $\begin{array}{l}\text { Choy et al. (2005); Araz e Ozkarahan (2007); W.-P. } \\
\text { Wang (2010); Keskin et al. (2010). }\end{array}$ \\
\hline $\begin{array}{l}\text { Selecionar a alternativa mais apropriada para contratos } \\
\text { de terceirização }\end{array}$ & Almeida (2001, 2007); Ordoobadi (2009b). \\
\hline
\end{tabular}




\section{Tabela 4 (continuação)}

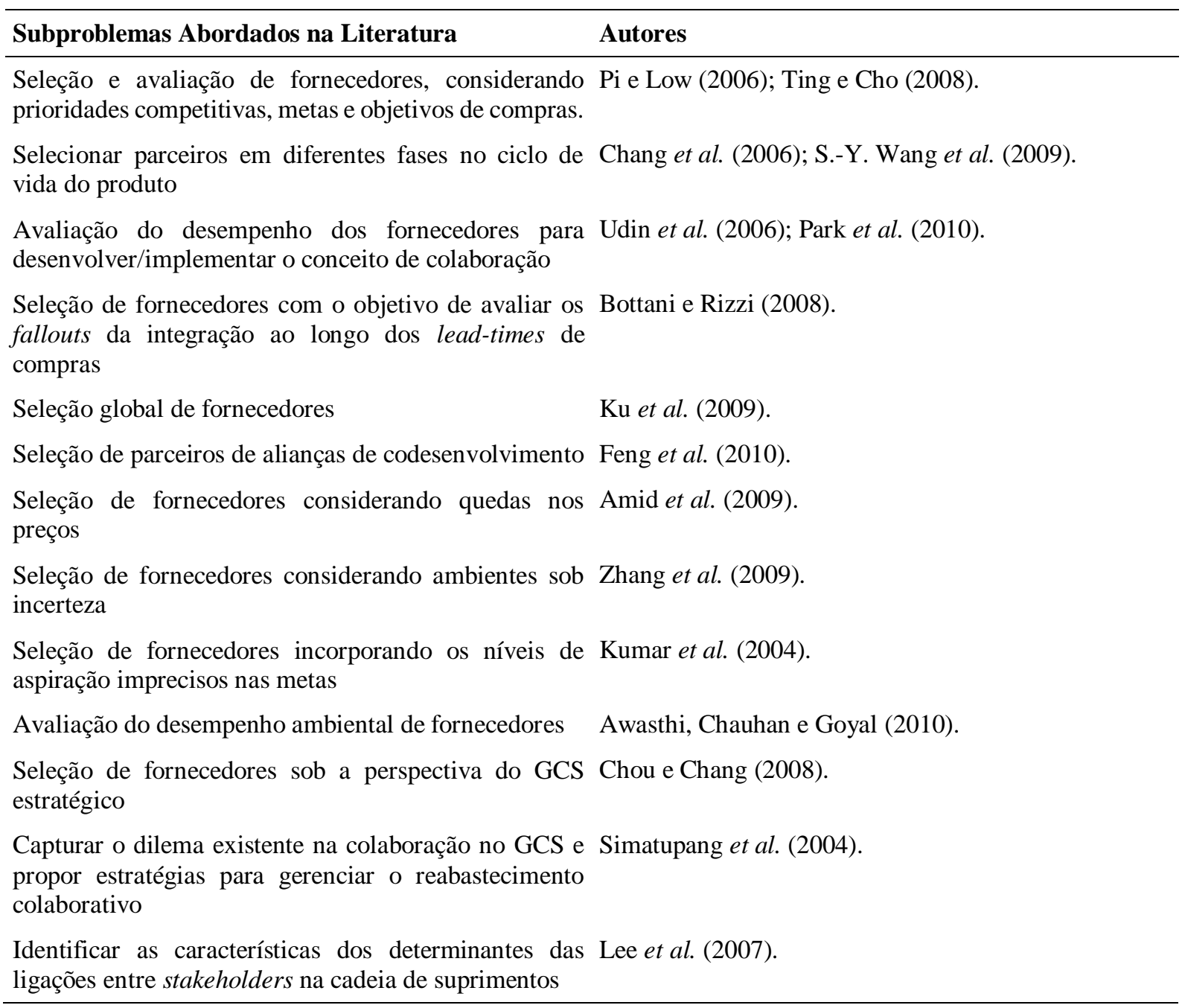

Conforme se observa na Tabela 4, a escolha de fornecedores possui vários subproblemas, além da questão de seleção propriamente dita sob a perspectiva da atividade de compras. Ressalta-se também que podem ser escolhidos fornecedores para relacionamentos colaborativos a fim de compartilhar informações, atividades e/ou recursos para a compra de produtos ou componentes. Nessa situação, requerem-se critérios específicos ao contexto envolvido.

Adicionalmente, foram elaborados quadros que resumem os métodos que consideram múltiplos critérios utilizados. Verificou-se que estes normalmente são utilizados em combinação com outras abordagens, por conseguinte, pode ocorrer que um mesmo autor figure em mais de um quadro, pois se julgou importante apresentar os métodos e suas variações contendo as abordagens agregadas. Entre os principais métodos encontrados na literatura, estão os da abordagem do Critério Único de Síntese, provenientes da corrente americana de pensamento, conforme se demonstra na Tabela 5. 
Tabela 5

\section{Variações dos Métodos Provenientes da Abordagem do Critério Único de Síntese}

\begin{tabular}{|c|c|}
\hline MÉTODO & AUTORES \\
\hline Fuzzy preference programming (FPP), Analytic hierarchy process (AHP) & Mikhailov (2002). \\
\hline Taguchi loss function, Analytical hierarchy process (AHP) & Pi e Low (2006). \\
\hline $\begin{array}{l}\text { Analytical hierarchy process (AHP), Data envelopment analysis (DEA) e Neural } \\
\text { Network (NN) }\end{array}$ & Ha e Krishnan (2008). \\
\hline $\begin{array}{l}\text { Analytical hierarchy process (AHP) weighted, Fuzzy linear programming model } \\
\text { (AHP-FLP) }\end{array}$ & Sevkli et al. (2008). \\
\hline Analytical hierarchy process (AHP), multi-objective linear programming (MOLP) & Ting e Cho (2008). \\
\hline Fuzzy Analytic hierarchy process (FAHP) & Yang et al. (2008). \\
\hline $\begin{array}{l}\text { Analytic network process (ANP), Multiobjective mixed integer linear programming } \\
\text { (MOMILP) }\end{array}$ & Ustun e Demirtas (2008). \\
\hline Fuzzy analytic hierarchy process (FAHP), Fuzzy goal programming (FGP) & Ku et al. (2009). \\
\hline $\begin{array}{l}\text { Analytic network process (ANP), Fuzzy preference programming (FPP), Multi- } \\
\text { objective linear programming (MOLP) }\end{array}$ & Lin (2009). \\
\hline $\begin{array}{l}\text { Taguchi loss function, Analytical hierarchy process (AHP), Multi-choice goal } \\
\text { programming (MCGP) }\end{array}$ & Liao e Kao (2010). \\
\hline Analytical hierarchy process (AHP) & Park et al. (2010). \\
\hline Multi-Atribute Utility Theory (MAUT) & Almeida (2001). \\
\hline
\end{tabular}

Conforme é possível perceber na Tabela 5, os métodos provenientes da abordagem do Critério único de síntese foram utilizados por $26 \%$ dos artigos pesquisados. É visível também que os métodos AHP e ANP são bastante utilizados, o que pode ser explicado pela facilidade que apresentam na estruturação do problema (Almeida, 2011). Os métodos AHP e ANP lidam adequadamente com critérios quantitativos e qualitativos, já o MAUT é mais voltado a critérios quantitativos. Todos os métodos dessa abordagem permitem trade-offs entre critérios. Na Tabela 6 são discriminados os métodos multicritério da abordagem de sobreclassificação, proveniente da corrente francesa de pensamento.

Tabela 6

Variações dos Métodos Provenientes da Abordagem de Sobreclassificação

\begin{tabular}{lc}
\hline MÉTODO & AUTORES \\
\hline $\begin{array}{l}\text { Preference Ranking Organisation Method for Enrichment Evaluations } \\
\text { (PROMETHEE), Graphical Analysis for Interactive Assistance (GAIA), High- } \\
\text { dimensional sensitivity analysis }\end{array}$ & Dulmin e Mininno (2003). \\
$\begin{array}{l}\text { ELimination Et Choix Traduisant la Realité (ELimination and Choice Expressing } \\
\text { Reality) ELECTRE, com função utilidade }\end{array}$ & Almeida (2001, 2007). \\
$\begin{array}{l}\text { Fuzzy goal programming (FGP), Preference Ranking Organisation Method for } \\
\text { Enrichment Evaluations (PROMETHEE) }\end{array}$ & Araz et al. (2007). \\
$\begin{array}{l}\text { Preference Ranking Organisation Method for Enrichment Evaluations Sorting } \\
\text { (PROMSORT) }\end{array}$ & Araz e Ozkarahan (2007). \\
\hline
\end{tabular}

De acordo com a Tabela 6, os métodos PROMETHÉE e ELECTRE foram utilizados em 9\% dos artigos pesquisados. Entre os trabalhos que adotam os referidos métodos, alguns são integrados também 
a outras abordagens que os complementam. As variações da Lógica Fuzzy são também amplamente utilizadas nos artigos como abordagem complementar.

Neste caso, percebe-se que o método PROMETHÉE é mais utilizado, sendo o método ELECTRE citado apenas em um artigo. Os métodos provenientes dessa abordagem possuem uma agregação não aditiva, ou seja, não permitem a compensação entre critérios e são considerados, por alguns autores, como Vincke (1992) e Almeida (2011), como métodos mais realísticos, os quais assumem que nem sempre o decisor está apto ou deseja demonstrar a preferência por uma dada alternativa, ele pode afirmar que as alternativas são incomparáveis e requerer mais tempo ou informações para posteriormente tomar a decisão.

Na Tabela 7, elencam-se os demais métodos utilizados nos artigos pesquisados quanto ao problema de seleção e avaliação de fornecedores que não se encaixaram nas categorias anteriores.

Tabela 7

Variações dos Métodos Provenientes da Abordagem dos Métodos Interativos

\begin{tabular}{ll}
\hline MÉTODO & AUTORES \\
\hline $\begin{array}{l}\text { Case based supplier selection and evaluation system (CSSES), Hybrid inductive- } \\
\text { nearest neighbor CBR approach }\end{array}$ & Choy et al. (2005). \\
$\begin{array}{l}\text { Weighted linear programming (LP), Data envelopment analysis (DEA) } \\
\text { Economic evaluation, Taguchi loss function }\end{array}$ & $\mathrm{Ng}(2008)$. \\
Two-stage stochastic programming (SP), Chance-constrained programming (CCP) & Li e Zabinsky (2011). \\
\hline
\end{tabular}

Na Tabela 7 foram discriminados os métodos provenientes da abordagem dos métodos interativos, os quais normalmente são baseados na Programação Linear, incluem um raciocínio matemático como base, sendo possível que o decisor interaja e, no momento em que ao não concordar com o resultado, possa simular outro resultado, caracterizando a dinâmica da tentativa e erro. Cabe ressaltar que esses métodos abrangem critérios quantitativos e permitem uma racionalidade aditiva, ou seja, permitem trade-offs entre critérios. Percebe-se que também normalmente esses métodos são combinados a outras abordagens com o intuito de complementar suas capacidades. Na Tabela 8, são enumeradas as variações provenientes da Lógica Fuzzy, a qual não faz parte da abordagem AMD, no entanto comumente é utilizada conjuntamente aos métodos dessa abordagem.

Tabela 8

Variações de Utilização da Lógica Fuzzy

\begin{tabular}{ll}
\hline MÉTODO & AUTORES \\
\hline Fuzzy preference programming (FPP), Analytic hierarchy process (AHP) & Mikhailov (2002). \\
$\begin{array}{l}\text { Fuzzy mixed integer goal programming (f-MIGP_VSP) } \\
\text { Asymmetric fuzzy-decision making technique (AFDMT) }\end{array}$ & Kumar et al. (2004). \\
$\begin{array}{l}\text { Fuzzy linguistic quantifier guided order-weighted aggregation (FLQG-OWA) } \\
\text { Fuzzy goal programming (FGP), Preference Ranking Organisation Method for }\end{array}$ & Amid et al. (2006). \\
$\begin{array}{l}\text { Enrichment Evaluations (PROMETHEE) } \\
\text { Analytical hierarchy process (AHP) weighted, Fuzzy linear programming (FLP) }\end{array}$ & Araz et al. (2007). \\
$\begin{array}{l}\text { Cluster analysis (CA), Multicriteria decision aid (MCDA) techniques, Fuzzy logic } \\
\text { Fuzzy analytic hierarchy process (AHP) }\end{array}$ & Bottani e Rizzi (2008). \\
\hline
\end{tabular}




\section{Tabela 8 (continuação)}

\begin{tabular}{|c|c|}
\hline MÉTODO & AUTORES \\
\hline Fuzzy Simple multiattribute rating technique (SMART) & Chou e Chang (2008). \\
\hline $\begin{array}{l}\text { Fuzzy group decision making and linear programming (FGDMLP) Technique for } \\
\text { order performance by similarity to ideal solution (TOPSIS), Fuzzy linear } \\
\text { programming (FLP) }\end{array}$ & Razmi et al. (2009). \\
\hline Fuzzy analytic hierarchy (FAHP), Fuzzy goal programming (FGP) & Ku et al. (2009). \\
\hline Fuzzy set theory & Ordoobadi (2009b). \\
\hline Fuzzy weighted additive (FWA), Mixed integer linear programming (MILP) & Amid et al. (2009). \\
\hline $\begin{array}{l}\text { Intuitionistic fuzzy group decision making (IFGDM), Technique for preference by } \\
\text { similarity to the ideal solution (TOPSIS) }\end{array}$ & $\begin{array}{l}\text { Boran et al. (2009), } \\
\text { Yayla et al. }(2012) \\
\text { Khaleie } \text { et al. }(2012) \text {. }\end{array}$ \\
\hline Quality Function Deployment (QFD), e Fuzzy Logic & Amin e Razmi (2009). \\
\hline $\begin{array}{l}\text { Ratio and multi-granularity linguistic scales, Fuzzy linguistic quantifier guided } \\
\text { ordered weighted aggregation (FLQGOWA) }\end{array}$ & $\begin{array}{l}\text { S.-Y. Wang et al. } \\
\text { (2009). }\end{array}$ \\
\hline Vague sets-based, Fuzzy multiple criteria decision-making (FMCDM) & Zhang et al. (2009). \\
\hline Weighted additive fuzzy multiobjective model & Amid et al. (2009). \\
\hline Fuzzy Adaptive ressonance theory (F-ART), Neural networks (NN) & Keskin et al. (2010). \\
\hline Fuzzy multiple attribute decision-making (FMADM) & Feng et al. (2010). \\
\hline $\begin{array}{l}\text { Fuzzy Sets Theory (FST), Vlse Kriterijumska Optimizacija I Kompromisno } \\
\text { Resenje/Multicriteria Otimization and compromise solution (VIKOR) }\end{array}$ & Sanayei et al. (2010). \\
\hline 2-Tuple fuzzy linguistic information & W.-P. Wang (2010). \\
\hline
\end{tabular}

Conforme pode ser percebido na Tabela 8, a Lógica fuzzy é adotada em $48 \%$ dos artigos pesquisados, cuja utilização é justificada pela capacidade de facilitar a captura das avaliações subjetivas dos decisores relacionadas aos critérios qualitativos e quantitativos considerados. O conceito fuzzy envolve imprecisão e conceitos vagos, os quais são muito comuns em critérios qualitativos, podendo ser entendido como uma situação na qual o decisor simplesmente não pode ou não consegue responder objetivamente a uma avaliação, atribuindo um número exato.

Assim, esta abordagem permite que o decisor escolha um intervalo de valores no lugar de um número exato, facilitando a captura dos julgamentos imprecisos provenientes do decisor. No entanto, como pode ser observado na Tabela 8, a lógica fuzzy é comumente integrada a outras abordagens para incrementar o seu ajuste ao problema estudado.

Os outros métodos que não se encaixaram nas classificações anteriores são apresentados na Tabela 9, como é o do TOC, Taguchi lossfunction e GAP analysis, os quais têm sido utilizados combinados a outras abordagens e foram utilizados em $6 \%$ dos artigos abrangidos, no entanto, têm em comum a consideração de múltiplos critérios e alternativas no problema de seleção e avaliação de fornecedores. Alguns desses métodos foram incorporados a técnicas gerenciais, como é o caso do método GAP analysis incorporado ao Hybrid knowledge based collaborative SCM. Constatou-se que alguns autores combinam métodos já consolidados na literatura; e outros, por sua vez, criam novos métodos com intuito de torná-los mais realísticos e aumentar a aderência ao problema de decisão. 
Tabela 9

Outros Métodos

\begin{tabular}{ll}
\hline MÉTODO & AUTORES \\
\hline Theory of constraints (TOC) & Simatupang et al. (2004). \\
Taguchi loss function & Pi e Low (2005). \\
Hybrid knowledge based collaborative SCM (KBCSCM), GAP analysis & Udin et al. (2006). \\
\hline
\end{tabular}

Entre os critérios mais utilizados nos artigos analisados, destacaram-se 18 que foram citados em três ou mais trabalhos e apresentados na Tabela 1. Entretanto é importante ressaltar que outros 76 critérios foram identificados, os quais foram citados em menos de três artigos, e alguns deles são importantes no estabelecimento de alianças e relacionamentos colaborativos. Esses critérios são constantemente citados como essenciais no processo de seleção de fornecedores, desse modo, abrangendo todos os seus subproblemas, pelos autores Min e Mentzer (2004), Cohen e Roussel (2004), Simatupang, Wright e Sridharan (2004), Bozarth e Handfield (2008) e Lambert (2008), no entanto não são amplamente utilizados. Vale ressaltar que estes autores abordam a temática de alianças estratégicas com fornecedores, e não o contexto multicritério, por isso, não compõem a Tabela 3.

Neste sentido, podem ser destacados os critérios: Comprometimento do comprador; co-design, resolução de conflitos; cooperação; correspondência de metas; incentivos; inovação; previsão de demanda interativa; comunicação interorganizacional; capacidade JIT; ação conjunta; proximidade do relacionamento; iniciativa e comprometimento dos fornecedores; programas de desenvolvimento de fornecedores e suporte da alta gerência. Esta baixa utilização justifica-se pela carência de trabalhos que envolvam o conceito de alianças e relacionamentos colaborativos, bem como a inclusão desses critérios em modelos multicritério para seleção de fornecedores pode ser considerada uma lacuna na literatura.

Quanto aos métodos mais utilizados nos artigos, percebe-se que a Lógica Fuzzy é abordada em quase metade dos artigos, o que pode ser explicado pela capacidade que esta abordagem tem de modelar os processos subjetivos de avaliação do decisor, convertendo tais avaliações em escalas de números fuzzy a fim de incorporar a hesitação do decisor em estabelecer valores devido a informações imprecisas, dados incertos ou maldeterminados. No entanto, dificilmente, a Lógica Fuzzy é abordada individualmente, e sim em conjunto com algum outro método com o intuito de agregar capacidades, como é o caso da ordenação e classificação das alternativas, tarefas específicas dos métodos AMD.

Também, devem ser destacados os métodos AHP e ANP, que fazem parte da abordagem do critério único de síntese. Esses métodos foram utilizados em aproximadamente $25 \%$ dos artigos pesquisados; sua utilização é considerável tendo em vista a facilidade de aplicação do método; a interface que auxilia o decisor a decompor e estruturar o problema e os critérios em uma hierarquia; e, também, por existirem no mercado quatro softwares para sua aplicação.

No que se refere aos métodos da abordagem de sobreclassificação, representada pelos métodos PROMETHÉE e ELECTRE, constata-se que foram utilizados em $9 \%$ dos artigos pesquisados. Esses métodos possuem uma lógica não compensatória, o que, de acordo com Almeida (2011), propiciam resultados mais equilibrados, considerando que não se aceita que um fornecedor com um desempenho muito baixo, em um determinado critério, possa ser beneficiado na escolha se possuir outro critério com um desempenho muito alto.

Além disso, destaca-se que 9\% dos artigos analisados utilizaram os métodos provenientes da abordagem dos métodos interativos, baseados na modelagem matemática. Além disso, outros métodos individuais ou combinados, como a Taguchi loss function, Constraints theory e GAP analysis foram também utilizados em $6 \%$ dos artigos.

Conforme mencionado anteriormente, a depender do contexto que envolve a natureza dos critérios, racionalidade do decisor e subproblema, justifica-se a utilização de um método específico, bem 
como a sua combinação com outras abordagens com o intuito de complementar a capacidade de lidar mais adequadamente com o problema em questão. Outrossim, constatou-se que artigos que tratem de seleção de fornecedores para alianças estratégicas e para relacionamentos colaborativos são escassos. Apenas cinco trabalhos possuem correspondência direta com estes subproblemas, os quais podem caracterizar também uma lacuna na literatura.

Destaca-se também que os métodos individuais mais utilizados encontrados neste trabalho foram os métodos AHP e ANP e, quanto aos métodos combinados, destaca-se o AHP Fuzzy. No tocante aos critérios mais utilizados, encontrou-se que o critério Custo é o mais utilizado, seguido por Qualidade e, então, Entrega. Denota-se que o critério Custo continua o fator preponderante nas negociações e que critérios considerados soft factors, conforme Ellram (1990), possuem pouca utilização, pois seriam mais apropriados para a seleção de parceiros para alianças estratégicas e relacionamentos cooperativos e colaborativos, os quais podem envolver relacionamentos ganha-ganha, dedicados a: co-design, coprodução, desenvolvimento conjunto de propriedade industrial e $\mathrm{P} \& \mathrm{D}$ e compartilhamento de informações e de ativos tangíveis e intangíveis.

Esta baixa utilização pode, em parte, ser explicada devido a esses critérios abrangerem fatores qualitativos, que envolvem um grau de subjetividade considerável e que, muitas vezes, são considerados de difícil mensuração por diversos autores e, também, pelo fato de existirem poucos artigos que abordam o problema de seleção de fornecedores sob a perspectiva de alianças estratégicas, conforme pode ser percebido na Tabela 4, na qual é possível verificar que o maior foco do problema de seleção de fornecedores é concentrado sob a perspectiva da atividade de compras. Nesse sentido, autores como Ellram (1990), Min e Mentzer, (2004), Choy, Lee, Lau e Choy (2005), Araz e Ozkarahan (2007) e Feng, Fan e Ma (2010) corroboram a ideia de que a combinação de critérios quantitativos, como o custo, a critérios qualitativos e estratégicos é mais apropriada e desejável na escolha de parceiros para alianças estratégicas.

Ademais, percebeu-se que, no processo de tomada de decisão para seleção de fornecedores, a consideração de múltiplos critérios é essencial, desta forma, a utilização da abordagem AMD torna-se válida, pois dá suporte aos gestores na escolha das alternativas mais adequadas de forma estruturada e sistematizada. Assim, reduzem-se as decisões tomadas somente com base na experiência, que podem representar riscos e incertezas no abastecimento da empresa, gerar paradas de produção e, consequentemente, prejuízos para todos os parceiros.

No entanto cabe ainda ressaltar que não há consenso nos artigos pesquisados quanto a um melhor método ou abordagem a ser adotado e, neste sentido, destaca-se a importância da análise de decisões. Analisando-se os fatores destacados neste artigo, é possível indicar o método mais apropriado a cada contexto, extraindo da abordagem AMD o suporte necessário para uma tomada de decisão mais consistente e adequada ao problema de seleção de fornecedores.

\section{Considerações Finais}

O problema de seleção de fornecedores possui caráter estratégico tendo em vista que muitas empresas têm delegado parte dos seus processos aos parceiros de negócios. Desta forma, escolher o parceiro adequado para cada atividade, considerando-se os objetivos estratégicos deste tipo de decisão e seus critérios, é tarefa essencial no GCS. É importante ressaltar que a análise de decisões abrange a Pesquisa Operacional Hard (PO-hard) e também a Pesquisa Operacional Soft (PO-soft). A primeira é relacionada a problemas bem-estruturados, enquanto a segunda é destinada a tratar dos problemas malestruturados, como é o caso do SSP, por conseguinte, isso requer abordagens que considerem a imprecisão dos julgamentos do decisor, como propõe a abordagem AMD.

Os métodos AMD, além de possuírem uma base matemática, levam em consideração os insights dos decisores, fato que é percebido, pelos principais autores da área como, por exemplo Vincke (1992), 
Roy (1996), Ehrlich (1996) e Almeida (2011), como uma de suas principais vantagens. Os problemas são mal-estruturados e requerem uma interação maior do analista com o decisor, diferentemente dos métodos provenientes da PO-hard, os quais são essencialmente matemáticos e objetivos, não considerando tais insights. No entanto ressalta-se que os métodos AMD normalmente requerem o auxílio de um analista (especialista em métodos AMD), tendo em vista que muitos gestores não dominam ainda a técnica de aplicá-los, o que tem sido em parte solucionado pelo surgimento de softwares que envolvem etapas de interação com o decisor.

Conforme ressaltam Wernke e Bornia (2001), a aplicação de métodos multicritério apresenta diversas vantagens porque permite a abordagem de problemas complexos (mal-estruturados), os quais não são tratados adequadamente por métodos intuitivos-empíricos ou puramente matemáticos, além de conferir ao processo de tomada de decisão maior transparência quando comparado a métodos monocritério. Campos e Almeida (2006) destacam a facilidade de uso e compreensão pelos decisores no processo principalmente quando os métodos AMD possuem etapas interativas e dispõem de ferramenta computacional. Cho e Cho (2008) enfatizam que os métodos multicritério fornecem uma estrutura abrangente para resolver problemas de decisão pela capacidade de quantificar julgamentos subjetivos dos decisores.

Cabe, todavia, ressaltar algumas limitações da presente pesquisa: a pesquisa limitou-se às bases ISI Web of Knowledge e Scopus; não considerou a revisão de métodos provenientes da PO-hard; tampouco os métodos da PO-soft utilizados exclusivamente para a estruturação de problemas de decisão, mas sim aqueles que abrangem critérios qualitativos e quantitativos. Também, não foi escopo desta pesquisa a aplicação de tais métodos, e sim o levantamento dos critérios, subproblemas e métodos mais abordados.

A presente pesquisa contribui ao indicar lacunas na literatura, as quais podem ser desenvolvidas em estudos futuros, tais quais: (a) incorporação de soft factors em modelos multicritério, os quais são mais adequados em relacionamentos colaborativos e alianças estratégicas; (b) o estudo do problema de seleção de fornecedores sob a perspectiva de parcerias e relacionamentos colaborativos; e, (c) a proposição de modelos gerenciais que abranjam uma estrutura de decisão sistematizada, envolvendo a abordagem AMD. Ademais, contribui ao apresentar aos gestores as possíveis abordagens a serem utilizadas neste tipo de decisão, a depender do contexto envolvido.

Outrossim, sugere-se que futuramente sejam desenvolvidos estudos focados no problema de seleção de fornecedores sob a perspectiva de alianças estratégicas, além de artigos que abordem a inclusão dos critérios conhecidos como soft factors propostos por Ellram (1990) e abordados por: Min e Mentzer (2004), Cohen e Roussel (2004) e Simatupang et al. (2004) - em modelos multicritério. Também se sugere o desenvolvimento de estudos que envolvam a proposição de modelos de decisão os quais incluam a decisão de escolha de parceiros para alianças estratégicas utilizando a abordagem AMD.

\section{Agradecimentos}

Este trabalho teve suporte parcial da Capes (Projeto Pró-engenharias).

\section{Referências}

Almeida, A. T. de (2001). Multicriteria decision making on maintenance: spares and contracts planning. European Journal of Operational Research, 129(2), 235-241. doi: 10.1016/S03772217(00)00220-4 
Almeida, A. T. de (2007). Multicriteria decision model for outsourcing contracts selection based on utility function and ELECTRE method. Computers \& Operations Research, 34(12), 3569-3574. doi: $10.1016 /$ j.cor.2006.01.003

Almeida, A. T. de (2011). O conhecimento e o uso de métodos multicritério de apoio a decisão. Recife: Editora Universitária da UFPE.

Amid, A., Ghodsypour, S. H., \& O’Brien, C. (2006). Fuzzy multiobjective linear model for supplier selection in a supply chain. International Journal of Production Economics, 104(2), 394-407. doi: 10.1016/j.ijpe.2005.04.012

Amid, A., Ghodsypour, S. H., \& O'Brien, C. (2009). A weighted additive fuzzy multiobjective model for the supplier selection problem under price breaks in a supply chain. International Journal of Production Economics, 121(2), 323-332. doi: 10.1016/j.ijpe.2007.02.040

Amid, A., Ghodsypour, S. H., \& O’Brien, C. (2011). A weighted max-min model for fuzzy multiobjective supplier selection in a supply chain. International Journal of Production Economics, 131(1), 139-145. doi: 10.1016/j.ijpe.2010.04.044

Amin, S. H., \& Razmi, J. (2009). An integrated fuzzy model for supplier management: a case study of ISP selection and evaluation. Expert Systems with Applications, 36(4), 8639-8648. doi: 10.1016/j.eswa.2008.10.012

Antunes, C., \& Alves, M. (2012, setembro). Programação linear multiobjetivo-métodos interativos e software. Anais do Congreso Latino-Iberoamericano de Investigación Operativa, Rio de Janeiro, RJ, Brasil, 16.

Araz, C., Ozfirat, P. M., \& Ozkarahan, I. (2007). An integrated multicriteria decision-making methodology for outsourcing management. Computers \& Operations Research, 34(12), 37383756. doi: 10.1016/j.cor.2006.01.014

Araz, C., \& Ozkarahan, I. (2007). Supplier evaluation and management system for strategic sourcing based on a new multicriteria sorting procedure. International Journal of Production Economics, 106(2), 585-606. doi: 10.1016/j.ijpe.2006.08.008

Awasthi, A., Chauhan, S. S., \& Goyal, S. K. (2010). A fuzzy multicriteria approach for evaluating environmental performance of suppliers. International Journal of Production Economics, 126(2), 370-378. doi: 10.1016/j.ijpe.2010.04.029

Boer, L. de, Labro, E., \& Morlacchi, P. (2001). A review of methods supporting supplier selection. European Journal of Purchasing \& Supply Management, 7(2), 75-89. doi: 10.1016/S09697012(00)00028-9

Boran, F. E., Genç, S., Kurt, M., \& Akay, D. (2009). A multi-criteria intuitionistic fuzzy group decision making for supplier selection with TOPSIS method. Expert Systems with Applications, 36(8), 11363-11368. doi: 10.1016/j.eswa.2009.03.039

Bottani, E., \& Rizzi, A. (2008). An adapted multi-criteria approach to suppliers and products selectionAn application oriented to lead-time reduction. International Journal of Production Economics, 111(2), 763-781. doi: 10.1016/j.ijpe.2007.03.012

Bozarth, C. C., \& Handfield, R. B. (2008). Introduction to operations and supply chain management. New Jersey: Pearson Education Inc.

Campos, V. R., \& Almeida, A. T. de (2006). Modelo multicritério de decisão para localização de Nova Jaguaribara com VIP analysis. Pesquisa Operacional, 26(1), 91-107. doi: 10.1590/S010174382006000100005 
Chang, S.-L., Wang, R.-C., \& Wang, S.-Y. (2006). Applying fuzzy linguistic quantifier to select supply chain partners at different phases of product life cycle. International Journal of Production Economics, 100(2), 348-359. doi: 10.1016/j.ijpe.2005.01.002

Chen, I. J., Paulraj, A., \& Lado, A. A. (2004). Strategic purchasing, supply management, and firm performance. Journal of Operations Management, 22(5), 505-523. doi: 10.1016/j.jom.2004.06.002

Cho, Y.-G., \& Cho, K.-T. (2008). A loss function approach to group preference aggregation in the AHP. Computers \& Operations Research, 35(3), 884-892. doi: 10.1016/j.cor.2006.04.008

Chou, S.-Y., \& Chang, Y.-H. (2008). A decision support system for supplier selection based on a strategy-aligned fuzzy SMART approach. Expert Systems with Applications, 34(4), 2241-2253. doi: 10.1016/j.eswa.2007.03.001

Choy, K. L., Lee, W. B., Lau, H. C. W., \& Choy, L. L. C. (2005). A knowledge-based supplier intelligence retrieval system for outsource manufacturing. Knowledge-Based Systems, 18(1), 117. doi: $10.1016 /$ j.knosys.2004.05.003

Cohen, S., \& Roussel, J. (2004). Strategic supply chain management: the five disciplines for Top Performance. New York: McGraw-Hill.

Cooper, M. C., Lambert, D. M., \& Pagh, J. D. (1997). Supply chain management: more than a new name for logistics. International Journal of Logistics Management, 8(1), 1-14. doi: $10.1108 / 09574099710805556$

Cronin, P., Ryan, F., \& Coughlan, M. (2008). Undertaking a literature review: a step-by-step approach. British Journal of Nursing, 17(1), 38-43.

Degraeve, Z., Labro, E., \& Roodhooft, F. (2000). An evaluation of vendor selection models from a total cost of ownership perspective. European Journal of Operational Research, 125(1), 34-58. doi: 10.1016/S0377-2217(99)00199-X

De-la-Torre-Ugarte-Guanilo, M. C., Takahashi, R. T., \& Bertolozzi, M. R. (2011). Revisão sistemática: noções gerais. Revista Escola de Enfermagem, 45(5), 1255-1261. doi: 10.1590/S008062342011000500033

Dickson, G. W. (1966). An analysis of vendor selection systems and decisions. Journal of Purchasing, 2(1), 5-17.

Dulmin, R., \& Mininno, V. (2003). Supplier selection using a multi-criteria decision aid method. Journal of Purchasing \& Supply Management, 9(4), 177-187. doi: 10.1016/S1478-4092(03)00032-3

Ehrlich, P. J. (1996). Modelos quantitativos de apoio às decisões. Revista de Administração de Empresas, 36(1), 33-41. doi: 10.1590/S0034-75901996000100006

Ellram, L. M. (1990). The supplier selection decision in strategic partnerships. Journal of Purchasing and Materials Management, 26(4), 8-14.

Feng, B., Fan, Z.-P., \& Ma, J. (2010). A method for partner selection of codevelopment alliances using individual and collaborative utilities. International Journal of Production Economics, 124(1), 159-170. doi: 10.1016/j.ijpe.2009.10.019

Govindan, K., Kannan, D., \& Haq, A. N. (2010). Analyzing supplier development criteria for an automobile industry. Industrial Management \& Data Systems, 110(1), 43-62. doi: $10.1108 / 02635571011008399$ 
Ha, S. H., \& Krishnan, R. (2008). A hybrid approach to supplier selection for the maintenance of a competitive supply chain. Expert Systems with Applications, 34(2), 1303-1311. doi: 10.1016/j.eswa.2006.12.008

Higgins, J. P. T., \& Green, S. (Eds.). (2011). Cochrane handbook for systematic reviews of interventions: version 5.1.0. Retrieved from www.cochrane-handbook.org

Keskin, G. A., Ilhan, S., \& Özkan, C. (2010). The fuzzy ART algorithm: a categorization method for supplier evaluation and selection. Expert Systems with Applications, 37(2), 1235-1240. doi: 10.1016/j.eswa.2009.06.004

Khaleie, S., Fasanghari, M., \& Tavassoli, E. (2012). Supplier selection using a novel intuitionist fuzzy clustering approach. Applied Soft Computing, 12(6), 1741-1754. doi: 10.1016/j.asoc.2012.01.017

Krajewski, L., Ritzman, L., \& Malhotra, M. (2009). Administração de produção e operações. São Paulo: Prentice Hall.

Ku, C.-Y., Chang, C.-T., \& Ho, H.-P. (2010). Global supplier selection using fuzzy analytic hierarchy process and fuzzy goal programming. Quality \& Quantity, 44(4), 623-640. doi: 10.1007/s11135009-9223-1

Kumar, M., Vrat, P., \& Shankar, R. (2004). A fuzzy goal programming approach for vendor selection problem in a supply chain. Computers \& Industrial Engineering, 46(1), 69-85. doi: 10.1016/j.cie.2003.09.010

Lambert, D. M. (2008). Supply chain management: processes, partnerships, performance. Sarasota: SCM Institute.

Larbani, M., Huang, C. Y., \& Tzeng, G. H. (2011). A novel method for fuzzy measure identification. International Journal of Fuzzy Systems, 13(1), 24-34.

Lee, C. W., Kwon, I.-W. G., \& Severance, D. (2007). Relationship between supply chain performance and degree of linkage among supplier, internal Integration, and customer. Supply Chain Management: An International Journal, 12(6), 444-452. doi: 10.1108/13598540710826371

Léger, J., \& Martel, J.-M. (2002). A multicriteria assignment procedure for a nominal sorting problematic. European Journal of Operational Research, 138(2), 349-364. doi: 10.1016/S03772217(01)00251-X

Li, L., \& Zabinsky, Z. B. (2011). Incorporating uncertainty into a supplier selection problem. International Journal of Production Economics, 134(2), 344-356. doi: 10.1016/j.ijpe.2009.11.007

Liao, C.-N., \& Kao, H.-P. (2010). Supplier selection model using Taguchi loss function, analytical hierarchy process and multi-choice goal programming. Computers \& Industrial Engineering, 58(4), 571-577. doi: 10.1016/j.cie.2009.12.004

Lin, R.-H. (2009). An integrated FANP-MOLP for supplier evaluation and order allocation. Applied Mathematical Modelling, 33(6), 2730-2736. doi: 10.1016/j.apm.2008.08.021

Mentzer, J. T., DeWitt, W., Keebler, J. S., Min, S., Nix, N. W., Smith, C. D., \& Zacharia, Z. G. (2001). Defining supply chain management. Journal of Business Logistics, 22(2), 1-25. doi: 10.1002/j.2158-1592.2001.tb00001.x

Mentzer, J. T., Min, S., \& Zacharia, Z. G. (2000). The nature of interfirm partnering in supply chain management. Journal of Retailing, 76(4), 549-568. doi: 10.1016/S0022-4359(00)00040-3 
Mikhailov, L. (2002). Fuzzy analytical approach to partnership selection in formation of virtual enterprises. Omega - The International Journal of Management Science, 30(5), 393-401. doi: 10.1016/S0305-0483(02)00052-X

Min, S., \& Mentzer, J. T. (2004). Developing and measuring supply chain management concepts. Journal of Business Logistics, 25(1), 63-99. doi: 10.1002/j.2158-1592.2004.tb00170.x

$\mathrm{Ng}$, W. L. (2008). An efficient and simple model for multiple criteria supplier selection problem. European Journal of Operational Research, 186(3), 1059-1067. doi: 10.1016/j.ejor.2007.01.018

Ordoobadi, S. M. (2009a). Application of Taguchi loss functions for supplier selection. Supply Chain Management: an International Journal, 14(1), 22-30. doi: 10.1108/13598540910927278

Ordoobadi, S. M. (2009b). Development of a supplier selection model using fuzzy logic. Supply Chain Management: an International Journal, 14(4), 314-327. doi: 10.1108/13598540910970144

Park, J., Shin, K., \& Chang, T.-W. (2010). An integrative framework for supplier relationship management. Industrial Management \& Data Systems, 110(4), 495-515. doi: $10.1108 / 02635571011038990$

Pi, W.-N., \& Low, C. (2005). Supplier evaluation and selection using Taguchi loss functions. International Journal Advanced Manufacturing Technology, 26(1/2), 155-160. doi: 10.1007/s00170-003-1975-5

Pi, W.-N., \& Low, C. (2006). Supplier evaluation and selection via Taguchi loss functions and an AHP. International Journal Advanced Manufacturing Technology, 27(5/6), 625-630. doi: 10.1007/s00170-004-2227-z

Razmi, J., Songhori, M. J., \& Khakbaz, M. H. (2009). An integrated fuzzy group decision making/fuzzy linear programming (FGDMLP) framework for supplier evaluation and order allocation. International Journal Advanced Manufacturing Technology, 43(5/6), 590-607. doi: 10.1007/s00170-008-1719-7

Roy, B. (1996). Multicriteria methodology for decision aid. Netherlands: Kluver Academic Publishers.

Sanayei, A., Mousavi, S. F., \& Yazdankhah, A. (2010). Group decision making process for supplier selection with VIKOR under fuzzy environment. Expert Systems with Applications, 37(1), 24-30. doi: 10.1016/j.eswa.2009.04.063

Sevkli, M., Koh, L. C. S., Zaim, S., Demirbag, M., \& Tatoglu, E. (2008). Hybrid analytical hierarchy process model for supplier selection. Industrial Management \& Data Systems, 108(1), 122-142. doi: $10.1108 / 02635570810844124$

Simatupang, T. M., Wright, A. C., \& Sridharan, R. (2004). Applying the theory of constraints to supply chain collaboration. Supply Chain Management: an International Journal, 9(1), 57-70. doi: $10.1108 / 13598540410517584$

Ting, S.-C., \& Cho, D. I. (2008). An integrated approach for supplier selection and purchasing decisions. Supply Chain Management: an International Journal, 13(2), 116-127. doi: 10.1108/13598540810860958

Udin, Z. M., Khan, M. K., \& Zairi, M. (2006). A collaborative supply chain management: Part 2 - the hybrid KB/gap analysis system for planning stage. Business Process Management Journal, 12(5), 671-687. doi: 10.1108/14637150610691064

Ustun, O., \& Demirtas, E. A. (2008). An integrated multi-objective decision-making process for multiperiod lot-sizing with supplier selection. Omega - The International Journal of Management Science, 36(4), 509-521. doi: 10.1016/j.omega.2006.12.004 
Velamuri, V. K., Neyer, A.-K., \& Möslein, K. M. (2011). Hybrid value creation: a systematic review of an evolving research area. Journal für Betriebswirtschaft, 61(1), 3-35. doi: 10.1007/s11301-011$0070-5$

Vincke, P. P. (1992). Multicriteria decision-aid. England: John Wiley \& Sons.

Wang, S.-Y., Chang, S.-L., \& Wang, R.-C. (2009). Assessment of supplier performance based on product-development strategy by applying multi-granularity linguistic term sets. Omega - The International Journal ou Management Science, 37(1), 215-226. doi: 10.1016/j.omega.2006.10.003

Wang, W.-P. (2010). A fuzzy linguistic computing approach to supplier evaluation. Applied Mathematical Modelling, 34(10), 3130-3141. doi: 10.1016/j.apm.2010.02.002

Weber, C. A., Current, J. R., \& Benton, W. C. (1991). Vendor selection criteria and methods. European Journal of Operational Research, 50(1), 2-18. doi: 10.1016/0377-2217(91)90033-R

Webster, J., \& Watson, R. T. (2002). Analyzing the past to prepare for the future: writing a literature review. MIS Quarterly, 26(2), xiii-xxiii.

Wen, J., Li, S., Lin, Z., Hu, Y., \& Huang, C. (2012). Systematic literature review of machine learning based software development effort estimation models. Information and Software Technology, 54(1), 41-59. doi: 10.1016/j.infsof.2011.09.002

Wernke, R., \& Bornia, A. C. (2001). A contabilidade gerencial e os métodos multicriteriais. Revista de Contabilidade e Finanças, 12(25), 60-71. doi: 10.1590/S1519-70772001000100004

Wong, A., Tjosvold, D., \& Zhang, P. (2005). Developing relationships in strategic alli- ances: commitment to quality and cooperative interdependence. Industrial Marketing Management, 34(7), 722-73. doi: 10.1016/j.indmarman.2004.12.007

Yang, J. L., Chiu, H. N., Tzeng, G.-H., \& Yeh, R. H. (2008). Vendor selection by integrated fuzzy MCDM techniques with independent and interdependent relationships. Information Sciences, 178(21), 4166-4183. doi: 10.1016/j.ins.2008.06.003

Yayla, A. Y., Yildiz, A., \& Ozbek, A. (2012). Fuzzy TOPSIS method in supplier selection and application in the garment industry. Fibres \& Textiles in Eastern Europe, 20(4), 20-23.

Zhang, D., Zhang, J., Lai, K.-K., \& Lu, Y. (2009). An novel approach to supplier selection based on vague sets group decision. Expert Systems with Applications, 36(5), 9557-9563. doi: 10.1016/j.eswa.2008.07.053

\section{Dados do Autor}

